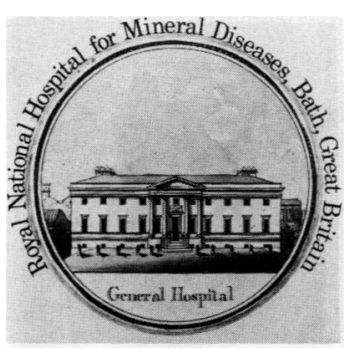

\title{
Management of the antiphospholipid syndrome
}

\author{
Munther A Khamashta, Tim Wallington
}

In 1987 Harris et $a l^{1}$ proposed that the combination of clinical features including both venous and arterial occlusive events, recurrent spontaneous abortions, and thrombocytopenia with antiphospholipid antibodies, identified as moderate to high titres of IgG or IgM anticardiolipin antibody or the lupus anticoagulant, should be termed the 'antiphospholipid syndrome'. Lupus anticoagulant, which was described in the 1950 s by Conley and Hartman, ${ }^{2}$ was first associated with thrombotic events by Bowie et al in 1963. ${ }^{3}$ Subsequently, antiphospholipid antibodies have been recognised to encompass a spectrum of antibody specificities which has been associated with an expanding range of clinical manifestations (table).

\section{Case reports}

The following cases illustrate some of the major clinical features of the antiphospholipid syndrome and the challenges posed to treatment.

\section{CASE NO 1}

A 60 year old white man, a retired engineer, presented at Southmead Hospital, Bristol in October 1990 as an emergency with unremitting severe parietal headache radiating to the occiput, which was associated with flashing lights, diplopia, and vomiting. There had been recent weight loss, general malaise, and loss of concentration. His general practitioner had found hypertension.

For two years he had had generalised musculoskeletal pain, which had been diagnosed at the Royal National Hospital for Rheumatic Diseases in September 1989 as fibromyalgia. In January 1990 he had severe vertigo accompanied by tinnitus. Meniere's disease was diagnosed and treated with betahistine. At that time brain stem evoked potentials were normal. For several months,

Features of the 'antiphospholipid syndrome'

\begin{tabular}{ll}
\hline $\begin{array}{l}\text { Major features } \\
\text { Venous and arterial occlusions } \\
\text { Thrombocytopenia }\end{array}$ & $\begin{array}{c}\text { Moderate to high titres of IgG } \\
\text { or IgM anticardiolipin } \\
\text { antibody, or both } \\
\text { Lupus anticoagulant }\end{array}$ \\
$\begin{array}{l}\text { Associated clinical features } \\
\text { Livedo reticularis }\end{array}$ & $\begin{array}{l}\text { Immune thrombocytopenic } \\
\text { purpura }\end{array}$ \\
$\begin{array}{l}\text { Heart valve lesions } \\
\text { Multistroke dementia } \\
\text { Chorea }\end{array}$ & \\
\hline
\end{tabular}

however, he had had olfactory and visual hallucinations.

There was no other relevant medical history. $\mathrm{He}$ was a non-smoker and there were no additional risk factors for cardiovascular disease.

On examination his speech was rather slow and he tended to get muddled. Formal neuropsychological testing, which included the Wechsler Adult Intelligence Scale, showed a marked memory defect. There was widespread livedo reticularis on his arms and legs. His blood pressure was $180 / 130$ but there was no papilloedema or retinopathy and the cardiovascular and neurological systems were otherwise normal.

Results of laboratory tests were as follows: haemoglobin $120 \mathrm{~g} / \mathrm{l}$; white blood cell count $5.9 \times 10^{9} / 1$, normal differential; platelets $105 \times 10^{9} / 1$; plasma viscosity $2 \cdot 13 \mathrm{mPa} \cdot \mathrm{s}$ (normal range 1.5-1.72); normal urea, electrolytes, and biochemistry. Urine analysis was normal. There was a mild hypergammaglobulinaemia: IgG 21 g/l, IgA $6.3 \mathrm{~g} / \mathrm{l}$, IgM $3.3 \mathrm{~g} / \mathrm{l}$. Antinuclear antibodies were detected, $320 \mathrm{IU} / \mathrm{ml}$, and antibodies to dsDNA were present in a moderate titre, $142 \mathrm{IU} / \mathrm{ml}$. Antibodies to the ribonucleoprotein antigens, UIRNP, Sm, Ro, and La, were not detected. Moderate titres of IgG anticardiolipin antibody (24 GPL units) and IgM anticardiolipin antibody (26 MPL units) were detected, however, but in the absence of a circulating anticoagulant. Serum complement concentrations were normal. A lumbar puncture produced clear cerebrospinal fluid, 130 red cells, one white blood cell, a normal glucose concentration but an increased protein concentration of $570 \mathrm{mg} / \mathrm{l}$. An electroencephalogram showed widespread increased slow wave activity. A brain computed tomography scan showed a doubtful area of reduced attentuation over the left basal ganglia, in keeping with a small infarct (figure A). A subsequent brain magnetic resonance imaging scan showed several high intensity shadows (figure B). A chest radiograph and electrocardiogram were normal and an echocardiogram was not obtained.

A diagnosis of systemic lupus erythematosus (SLE) with cerebral involvement was made. The hypertension was controlled with atenolol and nifedipine and, additionally, he was treated with oral prednisolone $60 \mathrm{mg}$ daily to which azathioprine was added. The prednisolone was subsequently reduced to an alternate day regimen and warfarin was added in January 1991. Since treatment there have been no further 

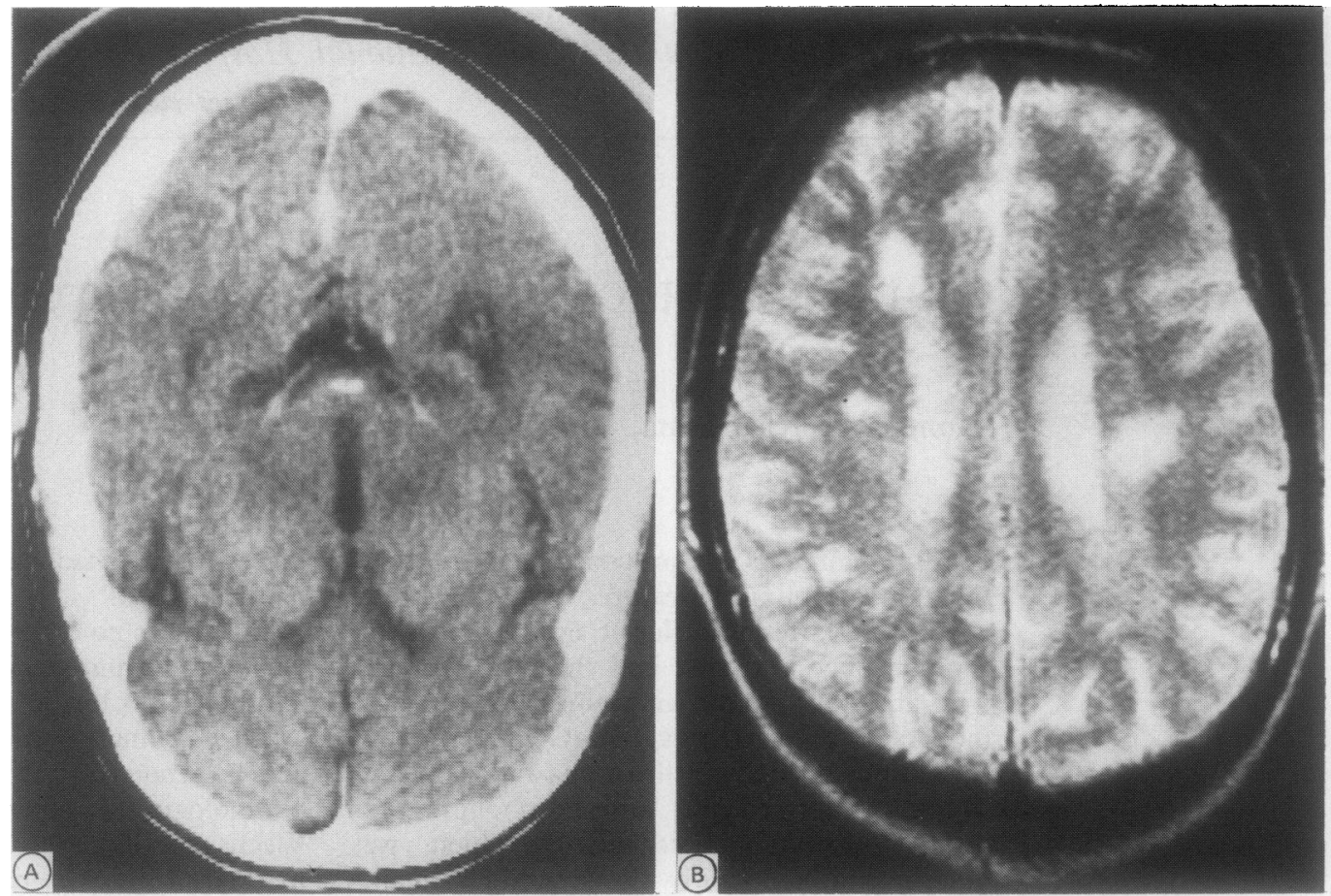

Computed tomographic scan of the brain $(A)$ showing a doubtful area of reduced attenuation over the left basal ganglia, whereas a magnetic resonance imaging scan $(B)$ performed shortly afterwards shows several high intensity shadows.

obvious cerebrovascular events and he has had no further hallucinations. Psychological testing has not yet been repeated, but he is now able to do the Telegraph crossword once more.

CASE NO 2

A 30 year old white woman first presented to the Royal National Hospital for Rheumatic Diseases in 1982 with a three month history of polyarthritis affecting the hands, wrists, and knees. The antinuclear antibody titre was weakly positive $(10 \mathrm{IU} / \mathrm{ml}$, homogeneous pattern) and there were no other abnormalities, but antiphospholipid antibodies were not specifically looked for. The symptoms responded to naproxen, the arthritis resolved completely within three months, and she was lost to follow up. Between 1981 and 1986 she had nine spontaneous abortions, all first trimester except for the initial abortion, which occurred at 16 weeks.

In December 1986 she presented acutely with fever, an expressive dysphasia, and weakness of the right side of her face and arm associated with paraesthesia. For three weeks previously there had been progressive malaise, throbbing headache, diffuse hair loss, and arthralgia. On examination there was facial butterfly erythema and livedo reticularis on the arms and legs. There was generalised lymphadenopathy and synovitis of the hands, wrists, and knees. The blood pressure was $150 / 90$, but there were no cardiological abnormalities. Results of a fundal examination were normal. There were upper motor neurone signs in the right arm, but the plantar responses were flexor.

The full blood count was normal, platelets $175 \times 10^{9} / 1$, as were the urea, electrolytes, and serum biochemistry. Urine analysis was normal. A lupus anticoagulant was detected (kaolin cephalin clotting time 74 seconds) together with high titres of IgG anticardiolipin antibody (134 GPL units). The antinuclear antibody titre was $20 \mathrm{IU} / \mathrm{ml}$ and low titres of anti-dsDNA were detected ( $50 \mathrm{IU} / \mathrm{ml})$. Antibodies to RNA binding proteins were not detected and complement concentrations were normal. A computed tomography brain scan showed attenuation in the left middle cerebral artery territory suggestive of an infarct.

A diagnosis of SLE with cerebral abnormality was made, and she was treated with an intravenous bolus of methylprednisolone $(1 \mathrm{~g})$ and cyclophosphamide $(750 \mathrm{mg})$. After two further pulses of methylprednisolone she was treated with daily oral prednisolone and low dose aspirin. She made a rapid recovery with resolution of the dysphasia and upper motor neurone signs. Mild but persistent hypertension was treated with a thiazide diuretic. During this period high titres of anticardiolipin antibody persisted but lupus anticoagulant activity disappeared only to return when the prednisolone dose was dropped below $10 \mathrm{mg}$ daily. In 1988 she became pregnant. The dose of prednisolone was increased empirically to $20 \mathrm{mg}$ daily and progress of the pregnancy was carefully monitored. After abnormal cardiotocograms a caesarean section was performed at 27 weeks and a live male infant weighing $630 \mathrm{~g}$ was delivered. Subsequently, the disease has been quiescent and treatment consists of low dose aspirin alone.

\section{Discussion}

The patients described here demonstrate two of the major clinical manifestations of the antiphospholipid syndrome-namely, cerebrovascular disease and recurrent abortions. As in case 2, the antiphospholipid syndrome can 
occur in association with SLE. Thrombotic events happen in about $30 \%$ of lupus patients with antiphospholipid antibodies and seem to be associated with high antibody titres and IgG, isotype. ${ }^{4}$ However, a high proportion of patients with the antiphospholipid syndrome do not fulfil the revised 1982 criteria for the classification of SLE. ${ }^{5}$ Some, like case 1, have a lupus-like disease, but an additional group, defined as having the 'primary' antiphospholipid syndrome, show no manifestations of SLE. ${ }^{6}$

Cerebrovascular disease, particularly transient ischaemic attacks and strokes, is the most common arterial manifestation of the antiphospholipid syndrome. As recently reviewed by Asherson et al, ${ }^{7}$ a broad range of central nervous system features is seen, including migrainous headaches. The association of livedo reticularis is well established and was first reported by Sneddon in $1965 .^{\circ}$ Although infarcts may be shown on a computed tomography scan (as in patient 2), magnetic resonance imaging has been shown to be more accurate in demonstrating lesions which are not always visible on a computed tomography scan. ${ }^{9}$ The worrying tendency in this syndrome is multiple cerebral infarcts complicated by progressive dementia. ${ }^{6}$ Clinically significant valve lesions have been associated with antiphospholipid antibodies and have been linked to cerebral disease. ${ }^{10}$ The possibility that valve lesions might serve as a source of emboli means that echocardiography is probably indicated in patients such as those described here, even without other signs of heart disease. ${ }^{11}$

Numerous case reports and reviews have demonstrated the association of recurrent spontaneous abortions and fetal death with antiphospholipid antibodies. ${ }^{12}$ The delivery of a term infant is extremely uncommon in a pregnancy complicated by a lupus anticoagulant. There is also a strong correlation between high titre IgG anticardiolipin antibody and pregnancy loss, and Lockshin et $a l^{13}$ have suggested that anticardiolipin antibody is the most sensitive marker for increased risk of pregnancy loss with a reported sensitivity of 0.85 and a specificity of 0.92 .

The consensus of opinion is that antiphospholipid antibodies have a pathogenetic role in the vasculopathy of the antiphospholipid syndrome, but the mechanism is unknown. Antiphospholipid antibodies comprise a broad range of specificities, with anticardiolipin antibody and lupus anticoagulant representing distinct subgroups. ${ }^{14}$ Proposed mechanisms to explain the thrombotic diathesis include increased platelet aggregation due to decreased production of prostacyclin through the effect of antiphospholipid antibodies on the vascular endothelium. ${ }^{15}$ Other groups have proposed inhibition of different plasma or platelet factors involved in the process of coagulation-thrombosis. ${ }^{16}$ The demonstration that a subset of antiphospholipid antibodies reacts with the complex of phospholipid and the serum protein, $\beta_{2}$ glycoprotein $I,{ }^{17}$ which is an inhibitor of the intrinsic coagulation pathway and binds platelet phospholipid, suggests a potent way in which antiphospholipid antibodies might predispose to a prothrombotic diathesis. Although this research may point to more targeted approaches to treatment, current treatment revolves around immunosuppression or anticoagulation or platelet inhibition, or a combination of these.

High risk patients are those with a history of features of the antiphospholipid syndrome or high titre anticardiolipin antibody or lupus anticoagulant, but as yet there is no general agreement among clinicians on the optimum treatment. Furthermore, controlled trials have not yet been done. Firstly, the controversy concerns whether or not prophylactic treatment is indicated. The Kingston Antiphospholipid Study (KAPS) Group was set up in part to answer this. In the meantime the consensus opinion is 'no' or at the most to use low dose aspirin. In the case of spontaneous abortions, although a high titre of IgG antiphospholipid antibodies is a major risk factor, there are several reports of normal pregnancies despite this serological finding. ${ }^{19}$ What is most important is regular assessment of intrauterine growth to predict fetuses at risk, which can be supplemented after about 18 weeks of pregnancy by Doppler flow studies of uteroplacental and umbilical artery circulation. ${ }^{20}$

In those with clinical features of the antiphospholipid syndrome various regimens have been reported, including corticosteroids, cytotoxic drugs, aspirin, dipyridamole, heparin, and warfarin as well as plasma exchange and high dose immunoglobulins. Many are single case reports and need to be treated with caution. The rationale for using corticosteroid treatment is suppression of lupus anticoagulant activity. ${ }^{21}$ Anticardiolipin antibody, however, is much more resistant to suppression. In 1983 Lubbe $e t$ $a l^{22}$ reported that prednisolone $40-60 \mathrm{mg} /$ day protected against fetal death. Subsequently, various combinations of high dose corticosteroids and low dose aspirin have been reported. This was the rationale for using prednisolone during pregnancy in case 2 . Such regimens, however, regularly produce severe cushingoid effects, hypertension, and diabetes, and the beneficial effects are questionable. ${ }^{23}$ Indeed, any trend towards better fetal survival may be due to the concurrent administration of low dose aspirin ${ }^{24}$ used to inhibit thromboxane $\mathrm{A}_{2}$ synthesis selectively, thereby theoretically preventing thrombosis of small vessels. Controlled trials are currently assessing aspirin in recurrent spontaneous abortions associated with antiphospholipid antibodies. Anticoagulation with subcutaneous heparin, which does not cross the placenta, is also currently being assessed in recurrent spontaneous abortions. ${ }^{25}$ The possibility of maternal osteoporosis is a limiting factor with this drug, although low molecular weight heparin, which has a long half life, can be given in a once daily regimen, and which may have less effect on bone, might be more promising.

In both cases described here an initial emphasis was put on high dose prednisolone in the treatment of the neurological manifestations. Although there was a rapid response of 
active lupus to corticosteroids in case 2 , there is no evidence that cerebrovascular manifestations of the antiphospholipid syndrome consistently respond to such treatment or that it prevents the development of recurrent strokes and multistroke dementia. Again, the management is controversial. Some favour conservative treatment with antiplatelet agents, such as low dose aspirin or dipyridamole, and others, anticoagulation. Management of recurrent peripheral venous or arterial thrombosis in association with antiphospholipid antibodies has traditionally been by anticoagulation with warfarin, and case reports suggest that indefinite treatment is required in this group of patients to prevent recurrences. ${ }^{26}$ Until results of controlled trials are available our policy is to introduce warfarin if there is evidence of progressive central nervous system abnormality.

A few studies have shown that hydroxychloroquine is of significant benefit in preventing thromboembolic disease postoperatively in high risk patients. ${ }^{27}$ Among its other actions, hydroxychloroquine is an inhibitor of phospholipase $A_{2}$ and therefore potentially affects platelet adhesion. Consequently, trials are being undertaken of this drug in the antiphospholipid syndrome.

It is hoped that the results of various controlled therapeutic trials in the antiphospholipid syndrome will indicate better ways of managing these difficult manifestations. At the same time more knowledge of the underlying pathophysiology and the precise role of antiphospholipid antibodies will lead to more targeted treatment.

1 Harris E N, Baguley E, Asherson R A, Hughes G R V Clinical and serological features of the "antiphospholipid syndrome" (APS) [abstract]. c. Hartman C. A haemorrhagic disorder caused by circulating anticoagulants in patients with disseminated lupus erythematosus. 7 Clin Invest 1953; 31: 621-3.

3 Bowie E J, Thompson J H, Pascuzzi C A, Owen C A Thrombosis in systemic lupus erythematosus despite circuThrombosis in systemic lupus erythematosus despite circu-

4 Harris E N, Chan J K H, Asherson R A, Aber V R, Gharavi A E, Hughes G R V. Thrombosis, recurrent fetal loss and thrombocytopenia. Predictive value of the anticardiolipi test. Arch Intern Med 1986; 146: 2153-6.

5 Tan E M, Cohen A S, Fries J F, et al. The 1982 revised criteria for the classification of systemic lupus erythematosus. Arthritis Rheum 1982; 25: 1271-7.
6 Asherson R A, Khamashta M A, Ordi-Ros J, et al. The "primary" antiphospholipid syndrome: major clinical and

7 Asherson R A, Khamashta M A, Gil A, et al. Cerebrovascular disease and antiphospholipid antibodies in systemic lupus disease and antiphospholipid antibodies in systemic lupus erythematosus, lupus-like disease, and the primary
phospholipid syndrome. Am $\mathcal{F}$ Med 1989; 86: 391-9.

8 Sneddon I B. Cerebral vascular lesion in livedo reticularis. $B$ F Dermatol 1965; 77: 180-5.

9 Vermess M, Bernstein R M, Bydder G M, Sreiner R E, Young I R, Hughes $G$ R V. Nuclear magnetic resonance NMR) imaging of the brain in systemic lupus erythematosus. $\mathcal{F}$ Comput Assit Tomogr 1982; 7: 461-7.

10 Asherson R A, Lubbe W F. Cerebral and valve lesions in systemic lupus erythematosus: association with anti-

11 Khamashta M A, Cervera R, Asherson R A, et al. Association of antibodies against phospholipids with heart valve disease of antibodies against phospholipids with heart valve disease

12 Lockshin M D, Qamar T, Ley R A. Anticardiolipin and related antibodies: thrombosis and fetal death. In: Scott related antibodies: thrombosis and fetal death. In: Scot tissue disorders. Oxford: Oxford Medical Publications, 1989: tissue disorder

13 Lockshin M D, Qamar T, Druzin M L, Goie S. Antibody to cardiolipin, lupus anticoagulant and fetal death. 7 Rheumatol 1987; 14: 259-62.

14 McNeil H P, Chesterman C N, Krilis S A. Anticardiolipin antibodies and lupus anticoagulants comprise separate antibody subgroups with different phospholipid binding characteristics. Br f Haematol 1989; 73: 506-13.

15 Carreras L O, Defreyn G, Machin S J, et al. Arterial thrombosis, intrauterine death and the lupus anticoagulant: detection of immunoglobulin interfering with prostacyclin fermation. Lancet 1985; i: 244-6.

16 Gonzalez-Buritica H, Khamashta M A, Hughes G R V. Is there a role for phospholipases in the anti-cardiolipin syndrome? Clin Exp Rheumatol 1988; 6: 341-2.

17 Galli M, Comfurius P, Maassen C, et al. Anticardiolipin antibodies (ACA) directed not to cardiolipin but to a plasm protein cofactor. Lancet 1990; 335: 1544-7.

18 McNeil H P, Simpson R J, Chesterman C N, Krilis S A. Anti-phospholipid antibodies are directed against a complex antigen that includes a lipid-binding inhibitor of coagulation: beta 2-glycoprotein I (apolipoprotein H). Proc Natl Acad Sci USA 1990; 87: 4120-4.

19 McHugh N J, Reilly P A, McHugh L A. Pregnancy outcome and autoantibodies in connective tissue disease. and autoantibodies in

20 Trudinger B J, Stewart G J, Cook C M, Connelly A, Exner T. Monitoring lupus anticoagulant-positive pregnancies with umbilical artery flow velocity wave forms. Obstet Gynecol 1988; 72: 215-8.

21 Lubbe W F, Liggins G C. Role of lupus anticoagulant and autoimmunity in recurent pregnancy loss. Semin Reprod Endocrinol 1988; 6: 181-90.

22 Lubbe W F, Butler W S, Palmer S J, Liggins G C. Fetal survival after prednioslone suppression of maternal lupus anticoagulant. Lancet 1983; 1: 1361-3.

23 Lockshin M D, Druzin M L, Qamar T. Prednisone does not prevent recurrent fetal death in women with antiphosphoprevent recurrent fetal death in women with antiphospho

24 Branch D W, Scott J R, Kochenour N K, Hershgold E. Obstetric complications associated with the lupus antiObstetric complications associated with the

25 Triplett D A, Harris N E. Antiphospholipid antibodies and reproduction. Am $\mathcal{F}$ Reprod Immunol 1989; 21: 123-31.

26 Asherson R A. Chan A K H, Harris E N, Gharavi A E, Asherson $R$ A, Chan A K H, Harris E N, Gharavi A E,
Hughes $G$, V. Anticardiolipin antibody, recurrent thrombosis, and warfarin withdrawal. Ann Rheum Dis 1985; 44: 823-5.

27 Johnson R, Orth M C, Charnley J. Hydroxychloroquine in prophylaxis of pulmonary embolism following hip arthroplasty. Clin Orthop 1979; 144: 174-9. 